\title{
A LINEAR TIME ALGORITHM FOR FINDING TREE 3-SPANNER ON 2-TREES *
}

\author{
B. S. Panda \\ Department of Mathematics \\ Indian Institute of Technology, Delhi \\ Hauz Khas, New Delhi 110 016, INDIA
}

\author{
Sajal K. Das \\ Department of Computer Science and Engineering \\ The University of Texas at Arlington, Arlington TX 76019, USA \\ das@cse.uta.edu
}

\begin{abstract}
A spanning tree $T$ of a graph $G$ is said to be a tree $t$-spanner if the distance between any two vertices in $T$ is at most $t$ times their distance in $G$. A graph that has a tree $t$-spanner is called a tree $t$-spanner admissible graph. The complexity of recognizing tree 3-spanner admissible graphs is still unknown. In this paper, a characterization of tree 3-spanner admissible 2-trees is presented. Linear time algorithms for recognizing tree 3-spanner admissible 2-trees and for constructing tree 3-spanners in such 2-trees are also proposed.
\end{abstract}

Keywords: Tree t-spanner, k-trees, Computational Complexity, Algorithms.

\section{Introduction}

A spanning subgraph $H$ of a graph $G$ is called a $t$-spanner if the distance between every pair of vertices in $H$ is at most $t$ times their distance in $G$. For a $t$-spanner $H$ of $G, t$ is called the stretch factor and $|E(H)|$, the number of edges in $H$, is called the size of the spanner. A $t$-spanner $H$ of $G$ is called a tree $t$-spanner if $H$ is a tree. The notion of $t$-spanner was intro-

\footnotetext{
*Part of the work was done when the first author was at the Department of Computer and Information Sciences, University of Hyderabad and was visiting the Department of Computer Science and Engineering, The University of Texas at Arlington. This work was supported by NASA Ames Research Center under Cooperative Agreement Number NCC 2-5395.
} 
cation networks, message routing, data analysis, motion planning, computational geometry, image processing, network design, and phylogenetic analysis (see $[1,2,3,9,10,13,17-21])$. The study of graph spanners has attracted many researchers and is currently an active area of research (see [4-9,12,15,19,22]).

The goal behind the notion of spanners is to find a sparse spanner $H$ of a given graph $G$ such that the distance between every pair of vertices in $H$ is relatively close to the corresponding distance in the original graph $G$. Therefore, one of the fundamental problems in the study of spanners is to find a minimum $t$-spanner, i.e., a $t$-spanner having minimum number of edges, for every fixed $\mathrm{i}$ integer $t \geq 1$. Unfortunately, the problem of finding a minimum $t$-spanner is NP-Hard for $t=2$ [18] and for $t \geq 3$ [8]. For a minimum $t$-spanner $H$ of $G$, $|E(H)| \geq|V(G)|-1$ with equality holding if and only if $H$ is a tree $t$-spanner, where $|V(G)|$ is the number of vertices of $G$. The problem of determining whether an arbitrary graph admits a tree $t$-spanner has been studied in detail.

Cai and Corneil [9] have shown that for a given graph $G$, the problem of deciding whether $G$ has tree $t$-spanner is NP-Complete for any fixed $t \geq 4$ and is linearly solvable for $t=1,2$. The status of the case $t=3$ is still open for arbitrary graphs. They have also observed that split graphs, co-graphs, and complement of bipartite graphs always have tree 3-spanner. Madanlal et al. [14] have shown that interval graphs and permutation graphs admit tree 3-spanner which can be constructed in linear time. They have also characterized regular bipartite graphs which admit tree 3-spanner. Recently, Brandstadt et al. [4] have shown that strongly chordal graphs and dually chordal graphs admit tree 4-spanner which can be computed in linear time.

Let $G[S], S \subseteq V$, be the induced subgraph of $G=(V, E)$ on $S$. A subset $C \subseteq V$ is said to be a clique if $G[C]$ is a maximal complete subgraph of $G$. A clique $C$ is called a $k$-clique if $|C|=k$. A 3-clique is called a triangle. A graph $G$ is called a $k$-tree if it can be obtained by the following recursive rules.

- Start with any $k$-clique as the basis graph. A $k$-clique is a $k$-tree.

- To any $k$-tree $H$ add a new vertex and make it adjacent to a $k$-clique of $H$, to form a $(k+1)$-clique.

Note that a tree is nothing but a 1-tree. A 2-tree is a $k$-tree for $k=2$.

In this paper, we, first, observe that 2-trees in general do not admit tree 3 -spanner. We, then, characterize those 2-trees that admit tree 3-spanner. We also present a linear time algorithm for recongnizing a tree 3-spanner admissible 2-tree and for constructing a tree 3-spanner of a tree 3-spanner admissible 2tree.

The main idea behind our characterization of tree 3-spanner admissible 2tree is the identification of forced edges, i.e, edges that will appear in any tree 3-spanner. We show that a 2 -tree $G$ admits a tree 3-spanner if and only if it does not contain any triangle containing all three forced edges. We show that this characterization and the technique for identifying forced edges enable us to recognize tree 3-spanner admissible 2-trees in linear time. A 2-tree $G$, as seen from the definition, consists of $|V(G)|-2$ triangles. We show that every 
triangle of a 2-tree $\mathrm{G}$ contributes at least one edge to every tree 3-spanner of $G$. Given a tree 3-spanner admissible 2-tree $G$, we employ a $D$-search (a search similar to the classical $B F S$ but differs from $B F S$ in that the next element to explore is the element most recently added to the list of unexplored elements) to search the triangles of $G$. Using this search, we explore all the triangles and keep on adding some edges of the triangles using certain rules to construct a spanning tree of $G$. We show that this tree is indeed a tree 3-spanner of G.

The rest of the paper is organized as follows. Section 2 presents some pertinent definitions and results. Section 3 presents the characterization of tree 3 -spanner admissible 2-trees. Section 4 presents the recognition algorithms of tree 3-spanner admissible 2-trees and an algorithm for constructing a tree 3spanner of a tree 3-spanner admissible 2-tree. The proof of correctness of these algorithms are presented in this section. Section 5 presents the complexity analysis of these algorithms. Finally, section 6 concludes the paper.

\section{Preliminaries}

For a graph $G=(V, E)$, let $N_{G}(\mathrm{v})=\{\mathrm{w} \in \mathrm{V} \mid v w \in \mathrm{E}\}$ be the set of neighbors of $v$. If $G\left[N_{G}(v)\right]$ is a complete subgraph of $G$, then $v$ is called a simplicial vertex of $G$. An ordering $\alpha=\left(v_{1}, v_{2}, \ldots, v_{n}\right)$ is called a perfect elimination ordering (PEO) of $G$ if $v_{i}$ is a simplicial vertex of $G\left[\left\{v_{i}, v_{i+1}, \ldots, v_{n}\right\}\right]$ for all $i, 1 \leq i \leq n$. Let $d_{G}(v)$ denote the degree of $v$ in $G$. Let $d_{G}(u, v)$ denote the distance from $u$ to $v$ in $G$. Unless otherwise stated the graph $G$ is assumed to be connected. A triangle $\{a, b, c\}$ is said to be simplicial triangle if one of its vertices is simplicial. An edge of a simplicial triangle is called simplicial if it is incident on a simplicial vertex of the triangle. A triangle is said to be interior if all of its edges are shared by at least two triangles. A triangle is said to be double interior if the triangle is interior and two of its adjacent triangles on different edges are interior. A triangle is said to be triple interior if the triangle is interior and its adjacent triangles on each of the three edges are also interior.

A graph is said to be chordal if every cycle of length at least four has a chord. $k$-trees are a subclass of chordal graphs. So, every $k$-tree has a PEO. A 2-tree is said to be a minimal triple interior ( respectively, double or single interior ) 2-tree if it contains a triple interior (respectively, double or single interior ) triangle but none of its proper subgraph contains a triple interior (respectively, double interior or interior) triangle.

Let $T r$ be a triangle of a minimal triple interior 2-tree. $T r$ is called an outer triangle if it contains a simplicial vertex. $T r$ is called an innermost triangle if it is triple interior. $T r$ is called inner triangle if it is neither innermost nor outer triangle. The multiplicity $M(e)$ of an edge $e$ is defined to be the number of triangle containing $e$. Let $T r$ be a triangle and $e_{1}, e_{2}, e_{3}$ be the three edges of $T r . T r$ is said to be one sided developing with respect to $e_{1}$ if either $M\left(e_{2}\right)=1$ and $M\left(e_{3}\right)>1$ or $M\left(e_{3}\right)=1$ and $M\left(e_{2}\right)>1$. Suppose $M\left(e_{2}\right)=1$ and $M\left(e_{3}\right)>1$. In this case, $e_{3}$ is said to be a developing edge of $T r$ with respect to $e_{1}$. $T r$ is said to be double side developing with respect to $e_{1}$ if $M\left(e_{2}\right)>1$ 
and $M\left(e_{3}\right)>1$. In this case, $e_{2}$ and $e_{3}$ are said to be developing edges of $T r$ with respect to $e_{1}$.

If $G-C$ is disconnected for a clique $C$ with components $H_{i}=\left(V_{i}, E_{i}\right)$, $1 \leq i \leq r, r \geq 2$, then $C$ is said to be a separating clique and $G_{i}=\mathrm{G}\left[\left(V_{i} \cup C\right)\right]$, is called a separated graph of $G$ with respect to $C, 1 \leq i \leq r$, and $r \geq 2$. Let $W\left(G_{i}\right)=\left\{v \in C \mid\right.$ there is a $w \in V_{i}$ with $\left.v w \in E(G)\right\}$. Cliques of $G$ other than $C$ which intersect $C$ are called relevant cliques of $G$ with respect to $C$. A relevant clique $C_{i}$ of $G_{i}$ for which $\left(C_{i} \cap C\right)=\mathrm{W}\left(G_{i}\right)$ is called a principal clique of $G_{i}$.

The existence of a principal clique of every separated graph of a chordal graph is guaranteed by the following result due to Panda et al [16].

Lemma 2.1 [16]: Every separated graph

$G_{i}$ of a chordal graph has a principal clique.

Let $H$ be a spanning subgraph of $G$. Since the $d_{H}(x, y) \leq t \times d_{G}(x, y)$ for every $x, y \in V(G)$ if and only if $d_{H}(x, y) \leq t$ for every edge $x y \in E(G)$, we have the following useful lemma.

Lemma 2.2 A spanning subgraph $H$ of $G$ is a $t$-spanner if and only if $d_{H}(x, y) \leq$ $t$ for every edge $x y \in E(G)$.

In view of Lemma 2.2, in the rest of the paper we assume that a spanning subgraph $H$ (a spanning tree $T$ ) of $G$ is a $t$-spanner (tree $t$-spanner) if $d_{H}(x, y) \leq t\left(d_{T}(x, y) \leq t\right)$ for every edge $x y \in E(G)$.

\section{Characterization of Tree 3-spanner Admissible 2-trees}

In this section, we present a characterization of tree 3-spanner admissible 2-tree. We do this by identifying the forced edges, i.e., edges which will appear in every tree 3-spanner.

Proposition 3.1: Let $G$ be a tree 3-spanner admissible 2-tree having at least 3 vertices. Then $G-v$ is a tree 3-spanner admissible 2-tree for every simplicial vertex $v$ of $G$.

Proof: Let $T$ be a tree 3-spanner of $G$. Let $v$ be any simplicial vertex of $G$. If $d_{T}(v)=1$, then $T-v$ is a tree 3-spanner for $G-v$. Suppose $d_{T}(v)=2$. Let $N_{G}(v)=\{x, y\}$. Then $v x, v y \in E(T)$ but $x y \notin E(T)$. Let $T_{1}=T-\{x v\} \cup\{x y\}$. Then, $T_{1}$ is a tree 3-spanner of $G-v$.

The following proposition, whose proof is omitted, follows from the above proposition.

Proposition 3.2: Let $G$ be a tree 3-spanner admissible 2-tree with at least 3 vertices and let $\alpha=\left(v_{1}, v_{2}, \ldots, v_{n}\right)$ be a PEO of $G$. Then $G\left[\left\{v_{i}, v_{i+1}, \ldots, v_{n}\right\}\right]$ is a tree 3-spanner admissible 2-tree for all $i, 1 \leq i \leq n-2$.

Note that every induced sub 2-tree of a 2-tree can be obtained by successively deleting simplicial vertices. Therefore, we have the following.

Proposition 3.3: Every induced sub 2-tree of a tree 3-spanner admissible 2-tree is tree 3-spanner admissible.

The following lemma implies that if a triangle in $G$ satisfies certain properties, then two out of its three edges are present in every tree 3-spanner of $G$. 
The proof of the following lemma, which uses the method of contradiction, can be found in the appendix.

Lemma 3.4: Let $G$ be a tree 3-spanner admissible 2-tree. If $G$ has an induced interior triangle, say $\{a, b, c\}$, then exactly two out of the three edges $a b, b c$, and $c a$ are present in every tree 3-spanner $T$ of $G$.

Let $G$ be a tree 3-spanner admissible 2-tree. Below, we show that every triangle $T r$ of $G$ contributes at least one edge to every tree 3-spanner $T$ of $G$. Lemma 3.5: Let $T$ be a tree 3-spanner of a 2-tree $G$. Then, $T$ contains at least one edge of every triangle $\{a, b, c\}$ of $G$.

Proof: (By contradiction) If $\{a, b, c\}$ is an interior triangle, then by Lemma $3.4, T$ contains two edges of $\{a, b, c\}$. If $\{a, b, c\}$ is a simplicial triangle, then $T$ must contain one of the simplicial edges of $\{a, b, c\}$. If $\{a, b, c\}$ is neither a simplicial triangle nor an interior triangle, then, wlg, $M(a b)>1$ and $M(a c)>$ 1. Since, $M(b c)=1, b, a, c$ is the only path of length 2 in $G$ from $b$ to $c$. Since, $T$ does not contain $b c, T$ contains a path of length at most 3 from $b$ to $c$. Since, the only path of length 2 from $b$ to $c$ is $b, a, c, T$ has no path of length 2 from $b$ to $c$ as $T$ does not contain $a b$ and $a c$. But, every path of length 3 from $b$ to $c$ contains either $a b$ or $a c$. So, there is no path of length at most 3 from $b$ to $c$ in $T$. This is a contradiction to the fact that $T$ is a tree 3-spanner of $G$. So, $T$ must contain one of the edges $a b, b c$, and $a c$.

Let $G$ be a tree 3-spanner admissible 2-tree. An edge $e$ of $G$ is said to be a forced edge if it belongs to every tree 3-spanner of $G$. An edge which is common to two interior triangle is called a strong edge. Strong edges are forced edges as shown below.

Lemma 3.6: Every strong edge of a tree 3-spanner admissible 2-tree is a forced edge.

Proof: Let $b c$ be a strong edge. So, $b c$ is common to two interior triangles, say $\{a, b, c\}$, and $\{b, c, d\}$ of a tree 3-spanner admissible 2-tree $G$. Let $T$ be a tree 3 -spanner of $G$. If possible, $T$ does not contain $b c$. By Lemma 3.4, two of the three edges $a b, b c$, and $c a$ are present in $T$ and two of the three edges $b c, c d$, and $b d$ are also present in $T$. Since, $T$ does not contain $b c, T$ contains the edges $a b, a c, c d$, and $b d$. Now, $a, b, d, c, a$ is a cycle in $T$. Hence a contradiction. So, $T$ contains $b c$. Hence, $b c$ is a forced edge.

Since, all the three edges of a triple interior triangles are strong edges, and hence forced edges by Lemma 3.6, we have the following corollary.

Corollary 3.7: Let $G$ be a 2-tree containing a triple interior triangle. Then $G$ can not have a tree 3-spanner.

A triangle having two forced edges is called a semi-forced triangle. Let $\{a, b, c\}$ be a semi-forced triangle having forced edges $a b$ and $b c$. A triangle $\{x, a, c\}$ is said to be dependent on the triangle $\{a, b, c\}$ if either $M(x a)>1$ or $M(x c)>1$. Suppose $\{x, a, c\}$ is dependent on the triangle $\{a, b, c\}$ and $M(x c)>1$. Then, the edge $x c$ of the dependent triangle is called a semistrong edge. The motivation behind introducing the concept of semi-strong edge is that semi-strong edges are also forced edges. 


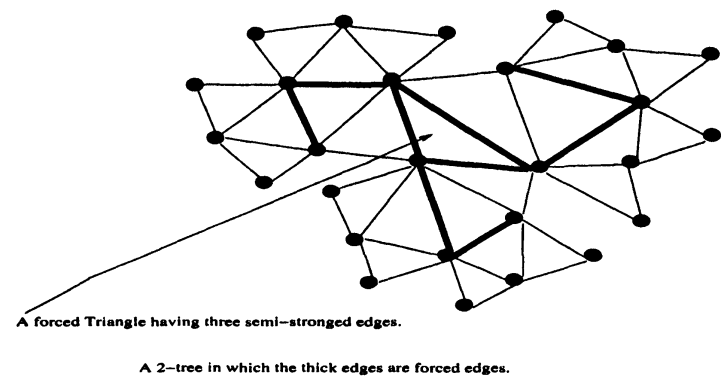

Figure 1. A 2-Tree without any triple interior triangle that has a triangle containing forced edges.

The following lemma whose proof can be found in the appendix, proves that semi-strong edges are forced edges.

Lemma 3.8: Every semi-strong edge of a tree 3-spanner admissible 2-tree $G$ is a forced edge.

We have seen earlier that if a 2-tree $G$ has a triple interior triangle, then it can not have a tree 3-spanner. A 2-tree may contain triangle consisting of forced edges that is not an interior triangle. Figure 1 contains a 2-tree which does not have any triple interior triangle but it has a triangle consisting of semi-strong edges. So, the graph does not have any tree 3-spanner.

The characterization theorem ( Theorem 3.11) for tree 3-spanner admissible 2-trees is proved using induction principle and the following lemma is a key in achieving that. The proof of the following lemma can be found in the appendix. Lemma 3.9: Let $G$ be a tree 3-spanner admissible 2-tree. Let $\{a, b, c\}$ be an interior triangle of $G$ and $H_{i}, 1 \leq i \leq 3$, be the connected components of $G-\{a, b, c\}$. Let $V\left(H_{2}\right)=\{e\}$ and $V\left(H_{3}\right)=\{f\}$. Let $d \in V\left(H_{1}\right)$ be such that $\{d, a, b\}$ is a triangle in $G_{1}=G\left[V\left(H_{1}\right) \cup\{a, b, c\}\right]$.

$(\alpha)$ If $\{d, a, b\}$ is an interior triangle in $G_{1}$, then $G_{1}$ contains two tree 3-spanners $T_{1}$ and $T_{2}$ such that $T_{1}$ contains the edges $a b$ and $a c$, and $T_{2}$ contains the edges $a b$ and $b c$.

( $\beta$ ) If $\{d, a, b\}$ is not an interior triangle of $G_{1}$ and ab is not a semi-strong edge, then $G_{1}$ has a tree 3-spanner $T_{3}$ containing any two edges of the three edges $a b, b c$, and $a c$.

The following lemma shows that a 2-tree, that does not have any interior triangle, admits a tree 3-spanner. The proof of the lemma indeed constructs a tree 3-spanner of a 2-tree that does not have any interior triangle. The proof of the following lemma can be found in the appendix.

Lemma 3.10: If a 2-tree is free from induced interior triangle, then it admits a tree 3-spanner.

Figure 2 contains a 2-tree which does not contain any interior triangle. So, by Lemma 3.10, it has a tree 3-spanner. The method which is employed in the proof of Lemma 3.10 is illustrated for the 2-tree $G$ of figure 2. The edges which 


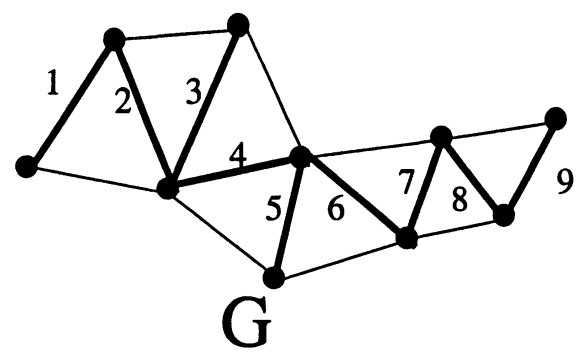

The thick edges form a tree 3-spanner.

Figure 2. Construction of a tree 3-spanner of a 2-tree with out having any interior triangle

are selected by the methods are the thick edges of $G$ and are numbered in the order they are selected. We employ a similar kind of method, which is more involved, to construct a tree 3-spanner of a tree 3-spanner admissible 2-tree.

A triangle of a 2-tree $G$ is said to be a strong triangle if each of its edges is either a strong edge or a semi-strong edge.

We are now in a position to characterize tree 3-spanner admissible 2-tree. The theorem is proved using induction principle and the proof is given in the appendix.

Theorem 3.11 (Characterization Theorem): A 2-tree $G$ admits a tree 3-spanner if and only if it does not contain a strong triangle as an induced subgraph.

\section{Algorithms and Proof of Correctness}

In this section, we first present a recognition algorithm for tree 3-spanner admissible 2-trees. Given a tree 3-spanner admissible 2-tree $G$, we next present an algorithm to find a tree 3-spanner of $G$. We also present the proof of correctness of these algorithms.

Algorithm Tree 3-spanner Recognition

Input: A 2-tree $G$;

Output: If $\mathrm{G}$ admits a tree 3-spanner then output " $\mathrm{G}$ admits a tree 3-spanner" and $E$, the set of forced edges else output "G has no tree 3-spanner".

1. Find all the triangles of G. $E=\phi$

2. For each strong edge $e$

Mark $e$ as forced edge.

$E=E \cup\{e\}$

3. Find all semi-strong edges, mark them as forced edges, and add them to $E$.

4. If $\mathrm{G}$ has a triangle containing all three edges in $E$, 
then output "G has no tree spanner" else output "G is a tree 3-spanner admissible 2-tree", and $E$. \}

Since, Algorithm tree 3-spanner Recognition checks for a strong triangle and declares that the 2-tree without having any strong triangle is tree 3-spanner admissible 2-tree, the proof of correctness follows from Theorem 3.11.

In view of the above, we have the following theorem.

Theorem 4.1: Algorithm tree 3-spanner recognition correctly recognize whether a 2-tree $T$ is tree 3-spanner admissible.

Next, we present an algorithm to construct a tree 3-spanner of a tree 3spanner admissible 2-tree $T$. We, then, prove the correctness of this algorithm.

The algorithm maintains a stack of edges, the current edge and a triangle containing the current edge as the current triangle. In every iteration, the stack is popped and the popped edge is made the current edge, and an unmarked triangle containing the current edge is made the current triangle. Based on the current edge and the current triangle, the algorithm pushes one or more edges to the stack. The algorithm also maintains two arrays, namely CUR and NUM to maintain the information of the triangles of $G . C U R[T r]=1$ if the triangle $T r$ is made current triangle at some iteration of the algorithm. Otherwise, $C U R[T r]=0$. NUM[Tr] represents the number when the triangle $T r$ was marked. The information stored in these arrays will be used for the proof of correctness of the algorithm. The exact rules for pushing the edges into the stack are given in the following algorithm.

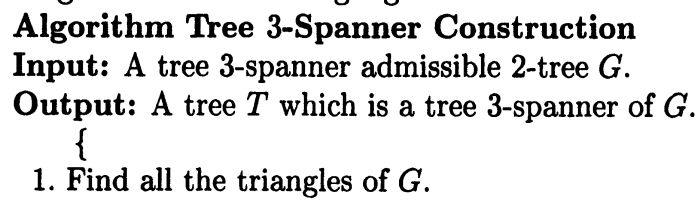

Output: A tree $T$ which is a tree 3-spanner of $G$.

1. Find all the triangles of $G$.

2. $Q=\phi$;

$T=\phi$;

$C U R[T r]=N U M[T r]=0$ for all triangles of $G$;

count $=1$;

Let $a b$ be a simplicial edge of $G$;

$\operatorname{Push}(Q, a b)$;

3. while $(Q \neq \phi) / *$ start of while loop $1 * /$

\{

$\mathrm{CE}=\mathrm{Pop}(\mathrm{S}) ; T=T \cup\{C E\}$;

while(there is any unmarked triangle, say $T r$, containing $C E$ )

/* start of while loop $2 *$ /

\{

$\mathrm{CT}=T r ; \mathrm{CUR}[\mathrm{Tr}]=1 ; \mathrm{NUM}[\mathrm{Tr}]=$ count $++;$ Mark $C T$;

Let the edges of $\operatorname{Tr}$ be $C E, e$ and $f$;

Case I: if ( $C T$ is one side developing w.r.t. $C E$ )

Let $C T$ be developing on $e$; 
if ( there is a unmarked triangle $T r^{\prime}=\{e, f, g\}$ such that $f$ and $g$ are

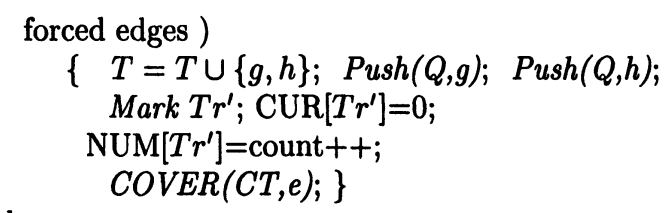

else

$$
\{T=T \cup\{e\} ; \operatorname{Push}(Q, e) ;\}
$$

w.r.t. $C E)$

Case II: if ( $C T$ is double side developing on $e$ and $f$

if ( either $e$ or $f$ is a forced edge )

$\{\mathrm{Wlg}$, let $e$ be a forced edge;

$T=T \cup\{e\} ; \operatorname{Push}(Q, e) ;$

$\operatorname{COVER}(C T, f) ;\}$

else

$\{T=T \cup\{e\} ;$

$\operatorname{Push}(Q, e)$;

$\operatorname{COVER}(C T, f) ;\}$

Case III: if $C T$ is zero-side developing

$T=T \cup\{e\} ;$

$\operatorname{Push}(Q, e)$;

\} $/ *$ end of while loop $2 * /$

\}

\} $/ *$ end of while loop $1 * /$

\section{Procedure COVER(CT,e)}

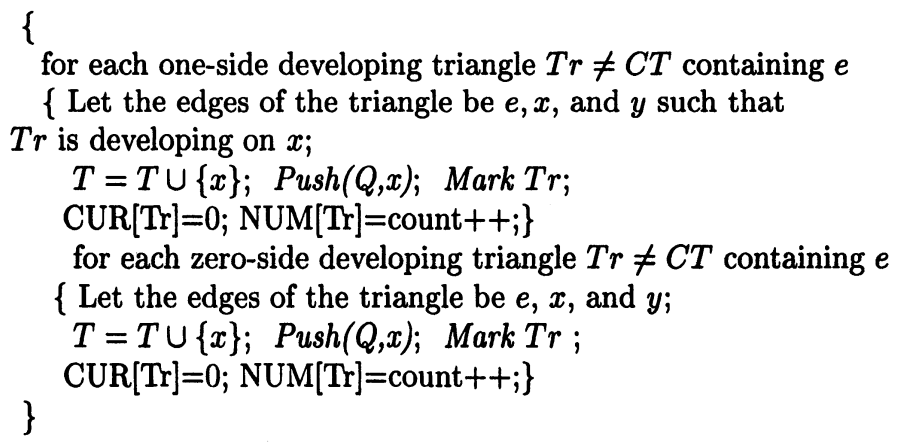

Next, we prove that Algorithm Tree 3-spanner Construction correctly constructs a tree 3-spanner of a tree 3-spanner admissible 2-tree. We do this by first showing that $T$, which is constructed by this algorithm, is a spanning tree of $G$, and then show that it is indeed a tree 3-spanner of G.

Theorem 4.2: Algorithm tree 3-spanner Construction produces a spanning tree $T$ of $G$. 
Proof: Note that Algorithm tree 3-spanner Construction consists of several iterations. In each iteration, a triangle is made current triangle, and based on whether it is one side developing, two side developing or zero side developing, certain triangles are marked. Let $T^{i}$ be the graph formed by the set of edges selected on or before ith iteration, and $S_{i}$ be the set of vertices spanned by the triangles which are marked on or before $i$ th iteration.

Claim: $T^{i}$ is a spanning tree of $\mathrm{G}\left[S_{i}\right]$ for each $i$.

Proof of Claim: Assume that the set of edges included in $T$ by the end of $(i-1)$ th iteration is a spanning tree of the set of vertices spanned by the set of triangles which are marked on or before $(i-1)$ th iteration. Let $C T=$ $\{C E, e, f\}$, and $C E$ be the current triangle, and current edge in $i$ th iteration, respectively. Edges are added to $T$ based on whether $C T$ is zero side, one side or two side developing.

Case I: $C T$ is zero-side developing.

In this case, only one edge $e$ is added to $T$. So, it is easy to see that our claim is true in this case.

Case II: $C T$ is developing one-side on $e$.

In this case, only one edge $e$ is added if there is no triangle $\{e, g, h\}$ containing two strong edges $g$ and $h$. In this case, our claim is easily seen to be true. Suppose, there is a triangle $\{e, g, h\}$, where $g$ and $h$ are strong edges. In such a situation, $g$ and $h$ are added to $T$ and $C T$ and $\{e, g, h\}$ are marked. Moreover, for all one side developing triangles $\{e, x, y\}$ on $x$, the edge $x$ is added and $\{e, x, y\}$ is marked and for all zero-side developing triangles $\left\{e, x_{1}, y_{1}\right\}, x_{1}$ is added to $T$ and $\left\{e, x_{1}, y_{1}\right\}$ is marked. So, $T^{i}$ forms a spanning tree on $G\left[S_{i}\right]$ in this case.

Case III: $C T$ is two-side developing.

Assume one of the edges $e$ and $f$ is a forced edge. Wlg, $e$ is a forced edge. In this case, $e$ is added to $T$ and $C T$ is marked. Also for all one-side developing triangles $\{f, g, h\}$ on $g$ and for all zero-side developing triangles $\{f, g, h\}$ the edge $g$ is added to $T$, and all such triangles are marked. So, $T^{i}$ is a spanning tree of $G\left[S_{i}\right]$ in this case.

Now, assume that neither $e$ nor $f$ is a forced edge. In this case as well, $e$ is added to $T$ and for all one-side developing triangles $\{f, g, h\}$ on $g$, the edge $g$ is added and $\{e, f, g\}$ is marked, and for all zero-side developing triangles $\left\{f, g_{1}, h_{1}\right\}$ the edge $g_{1}$ is added to $T$, and all such triangles are marked. So, $T^{i}$ is a spanning tree of $G\left[S_{i}\right]$ in this case.

So, our claim is true. Hence, $T$ is a spanning tree of $G$.

Next, we show that $T$ is a tree 3-spanner of $G$.

Theorem 4.3: Let $G$ be a tree 3-spanner admissible 2-tree. Then algorithm tree 3-spanner construction successfully constructs a tree 3-spanner of $G$.

Proof: By Theorem 4.2, the graph $T$ produced by the algorithm is a spanning tree of $G$.

Let $e \in E(G)$ and $e=x y$. If $e \in T$, then $d_{T}(x, y)=1$. So, assume that $e \notin T$. Let $T r$ be the triangle containing $e$ such that $N U M[T r]$ is minimum.

Case I: $C U R[T r]=1$. 
Let $T r=\{C E, e, f\}$. Since, $e \notin T, e \neq C E$. If $T r$ is zero-sided developing, then $f$ is added to $T$. So, $d_{T}(x, y)=2$. If $T r$ is one-side developing, then either $f$ is added or $g$ and $h$ are added to $T$, where either $\{e, g, h\}$ or $\{f, g, h\}$ is a triangle containing two forced edges $g$ and $h$. In this case, $d_{T}(x, y) \leq 3$. If $T r$ is two-side developing, then $d_{T}(x, y)=2$ as $f$ is added to $T$ in this case. So, $d_{T}(x, y) \leq 3$ if $C U R[T r]=1$.

Case II: $C U R[T r]=0$.

Let $\operatorname{Tr} 1$ be the current triangle when $\operatorname{Tr}$ was marked. So, $\operatorname{Tr} 1$ was either one-side developing or two-side developing. In either case, it can be seen using the arguments employed above that $d_{T}(x, y) \leq 3$.

So, $T$ is tree 3-spanner of $G$.

So, by Theorem 4.2 and Theorem 4.3, we have the following result.

Theorem 4.4: Algorithm tree 3-spanner construction correctly constructs a tree 3-spanner of a tree 3-spanner admissible 2-tree.

\section{Complexity Analysis}

In this section, we show that Algorithm Tree 3-spanner Recognition and Algorithm Tree 3-spanner Construction can be implemented in $O(n+m)$ time, where, $n$ and $m$ are the number of vertices, and number of edges of the input 2-tree.

Assume that the input graph $G$ which is a 2-tree is given in adjacency list representation. First we do some preprocessing and compute certain information which will make the implementation of the above mentioned algorithms easy.

First, find all the triangles of $G$. Since, cliques in chordal graphs can be found in $O(n+m)$ time [11], and cliques in 2-tree (which is a chordal graph) are triangles, all the triangles can be found in $O(n+m)$ time. Scan the adjacency lists and find a numbering of the edges of $G$. Modify the adjacency list of $G$ such that for each $v \in V, L(v)$, the adjacency list of $v$, contains an adjacent vertex, say, $w$, edge number of the edge $v w$ as obtained in the previous step, and a pointer to the next cell. This step takes $O(n+m)$ time. Next, number all the triangles and construct an array $T N$ of pointers such that $T N[i]$ contains the list of edges of the triangle having number $i$. This takes $O(n+m)$ time. Construct an array $A$ of pointers such that $A[i]$ contains the list of triangle containing the edge having number $i$. This can be done as follows. From the list of all triangles, construct a list of order pair by replacing a triangle, say $T r$, by $(e, T r),(f, T r)$, and $(g, T r)$, where $e, f$, and $g$ are the edges of $T r$. Now, sort this list in non-decreasing order on the first component. All the triangles containing an edge appear consecutively on this list. Since each edge has a unique number, bucket sort can be used to sort the above list. So, this takes $O(n+m)$ time. Now, from this sorted list, the array $A$ can be constructed in $O(n+m)$ time. Now, from the lists $T N$ and $A$, we can construct an array $N$ such that $N[i]=1$ if the triangle having number $\mathrm{i}$ is interior, else $N[i]=0$. This takes $O(n+m)$ time. Using the list $N$, it is easy to find all the strong edges of $G$ in $O(n+m)$ time. 
We, now, describe the implementation of Algorithm tree 3-spanner recognition. As discussed above, step 1 takes $O(n+m)$. Implementation of step 2 is straightforward once the list of interior triangles is given. Let $S_{i}, 0 \leq i \leq 3$, be the set of triangles having $i$ strong edges. Recall that, for each edge $\bar{e}, \bar{M}(e)$ is the number of triangles containing $e$. Then, step 3 can be implemented as follows:

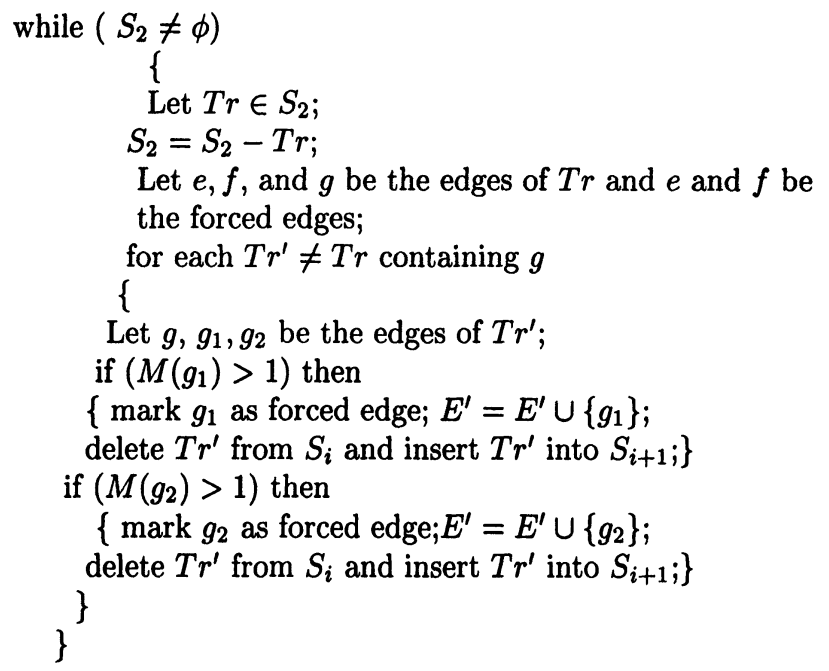

It is easy to see that this takes $O(n+m)$ time. Since, $S_{3} \neq \phi$ if and only if the 2-tree $G$ has a triangle consisting of three forced edges, implementation of step 4 is easy.

Since, 2-trees can be recognized in linear time [11], in view of the above and by Theorem 4.1 , we have the following theorem.

Theorem 5.1: Tree 3-spanner admissible 2-trees can be recognized in linear time.

Next, we show that Algorithm tree 3-spanner Construction can also be implemented in $O(n+m)$ time.

Algorithm tree 3-spanner starts by selecting a simplicial edge. This can be done by selecting an edge e with $A[e]=1$. This takes $O(n+m)$ time. Next, the algorithm marks the triangle containing the edge $e$. Whether the current triangle develops in one direction of two direction can be tested by checking the array $A$ for the edges of the triangle. The edge number of the edges can be found out by scanning the appropriate list of the modified adjacency list of $G$ obtained above. Again, whether a triangle is two-side developing, oneside developing or zero-side developing can be tested in $O(n+m)$ time for . all triangles. Now it is easy to see that other operations of Algorithm tree 3-spanner construction can be implemented in $O(n+m)$ time. So, algorithm tree 3-spanner construction takes $O(n+m)$ time. In view of the above and by Theorem 4.3, we have the following theorem. 
Theorem 5.2: Tree 3-spanner in a tree 3-spanner admissible 2-tree can be constructed in linear time.

\section{Conclusion}

In this paper, we have observed that 2-trees in general do not admit tree 3-spanner. We characterize those 2-trees that admit tree 3-spanner. We have also presented linear time algorithms for recognizing tree 3-spanner admissible 2-tree and for constructing a tree 3-spanner in a tree 3-spanner admissible 2tree. It would be interesting to study the tree 3-spanner problem on chordal graphs and in particular on $k$-trees for $k \geq 3$.

\section{References}

[1] I.Althöfer, G.Das, D.Dobkin, D. Joseph, and J. Soares, On sparse spanner of weighted graphs, Discrete Comput. Geom. 9 (1993), 81-100.

[2] J.P.Barthélemy and A. Guénoche, Trees and Proximity Representations, Wiley, New Yark, 1991.

[3] S.Bhatt, F.Chung, F.Leighton, and A. Rosenberg, Optimal simulation of tree machines, in "27th IEEE Foundations of Computer Science, Toronto, 1986, " pp. 274-282.

[4] A. Brandstadt, V. Chepoi, and F. Dragan, Distance approximating trees for chordal and dually chordal graphs, Journal of Algorithms, 30 (1999) 166-184.

[5] L.Cai and D.G. Corneil, Tree Spanners: an Overview, Congressus Numerantium 88 (1992), 65-76.

[6] L. Cai and J. M. Keil, Degree-Bounded Spanners, Parallel Processing Letters, 3(1993), 457-468.

[7] L. Cai and J. M. Keil, Spanners in Graphs of Bounded Degree, Networks, 24(1994),187-194.

[8] L.Cai, NP-completeness of minimum spanner problems, Disc. Appl. Math., 48 (1994), 187-194.

[9] L. Cai and D.G.Corneil, Tree Spanners, SIAM J. Discrete Ma th. 8 (1995) 359-387.

[10] L.P.Chew, There are planar graphs almost as good as the complete graph, J. Comput. Syst. Sci. 39 (1989), 205-219

[11] M. C. Golumbic, Algorithmic Graph Theory and Perfect Graphs. (Academic Press, New York, 1980).

[12] A.L.Liestman and T.C. Schermer, Grid Spanners, Networks, 23 (2) (1993) 123-133.

[13] A. L. Liestman and T. C. Shermer, Additive graph Spanner, Networks, 23 (1993), 343-364.

[14] M.S.Madanlal, G. Venkatesan, and C. Pandu Rangan, Tree 3-spanners on interval, permutation and regular bipartite graphs, Infor. Proc. Lett. 59 (1996) 97-102.

[15] G. Narasimhan, B. Chandra, D. Gautam, and J. Soares, New sparseness results on graph spanners, in 8th Annual ACM Symposium on Computational Geometry (1992) 192-201.

[16] B. S. Panda and S.P.Mohanty, Intersection graphs of vertex disjoint paths in a tree, Discrete Math. 146 (1995) 179-209.

[17] D. Peleg and J. D. Ullman, An optimal Synchronizer for the hypercube, proceedings of the 6th ACM Symposium on principles of Distributed computing, Vancouver (1987) 77-85.

[18] D. Peleg and E. Upfal, A trade off between space and efficiency for routing tables, Proceedings of the 20th ACM Symposium on Theory of Computing, Chicago (1988), 43-52.

[19] D. Peleg and A. A. Schäffer, Graph spanners. J. Graph Theory 13 (1989) 99-116.

[20] P.H.A.Sneath and R.R. Sokal, Numerical Taxonomy, San Francisco, 1973.

[21] D.L.Swofford and G.J.Olsen, Phylogeny reconstruction, in (D.M.Hills and C. Moritz, eds.), Molecular Systematics, pp. 411-501, Sinauer Associates, Sunderland, MA, 1990.

[22] G. Venkatesan, U.Rotics, M. Madanlal, J.A. Makowsky, and Pandu Rangan, Restrictions of minimum spanner problems, Information and computation, 136(2)(1997)143-164. 


\section{Appendix}

\section{Proof of Lemma 3.4:}

Proof:( By contradiction) Let $\{a, b, c, d, e, f\}$ be the vertices of an interior triangle $\{a, b, c\}$ such that $d$ is adjacent to $a$ and $b, e$ is adjacent to $b$ and $c$, and $f$ is adjacent to $a$ and $c$.

Case 1: None of the edges $a b, b c$ and $c a$ is present in $T$.

So, $d_{T}(a, b) \geq 2$ and $d_{T}(b, c) \geq 2$. Let $P(a, b)$ and $P(b, c)$ be the paths in $T$ from $a$ to $b$ and $b$ to $c$, respectively. If $P(a, b)$ and $P(b, c)$ have no edges in common, then clearly $d_{T}(a, c)>3$, a contradiction to the fact that $T$ is a tree 3-spanner of $G$. So, assume that $P(a, b)$ has an edge in common with $P(b, c)$. If $d_{T}(a, b)=d_{T}(b, c)=2$ and $x b$ is the common edge, then $\{a, b, c, x\}$ is a $K_{4}$, a complete graph on four vertices, which is a contradiction to the fact that $G$ is a 2-tree. So, either $d_{T}(a, b)>2$ or $d_{T}(b, c)>2$. If possible $d_{T}(a, b)=3$ and $d_{T}(b, c)=2$. Let $P(a, b)=a, x, y, b$ and $P(b, c)=b, y, c$. Now $a, x, y, b, a$ is a cycle of length 4. So, either $a y \in E(G)$ or $b x \in E(G)$. If $a y \in E(G)$, then $\{a, y, b, c\}$ is a $K_{4}$, which is a contradiction to the fact that $G$ is a 2-tree. If possible, $x b \in E(G)$. Now, $a, x, y, c, a$ is a cycle of length 4 . So, either $x c \in E(G)$ or $a y \in E(G)$. As we have seen $a y \in E(G)$ leads to a contradiction, so $x c \in E(G)$. Now $\{a, b, c, x\}$ is a $K_{4}$. Hence a contradiction.

So $d_{T}(a, b)=3$ and $d_{T}(b, c)=3$. Let $P(a, b)=a, x, y, b$. Suppose $P(b, c)$ has exactly one edge in common with $P(a, b)$. Since, $a b$ and $b x$ can not be present in $T, P(b, c)$ will be of the form $b, y, z, c$. Now, $d_{T}(a, c)>3$, which is a contradiction to the fact that $T$ is a tree 3-spanner of $G$. So, assume that $P(b, c)$ has two edges in common with $P(a, b)$. Let $P(b, c)=b, y, x, c$. Now, $b, y, x, c, b$ is a cycle of length 4 . So, either $x b \in E(G)$ or $c y \in E(G)$. If $b x \in E(G)$, then $\{a, b, c, x\}$ is a $K_{4}$, which is a contradiction to the fact that $G$ is a 2-tree. So, $c y \in E(G)$. Again, $a, x, y, b, a$ is a cycle of length 4. Since, $b x$ is not an edge of $G, a y \in E(G)$. Now, $\{a, b, c, y\}$ is a $K_{4}$, which is a contradiction.

Case 2: Exactly one of the edges $a b, b c$ and $c a$ is present in $T$.

$\mathrm{Wlg}, a b \in E(T)$. Now $d_{T}(b, c) \geq 2$. Suppose $d_{T}(b, c)=2$. Let $P(b, c)=$ $b, x, c$. Now, $d_{T}(f, a) \leq 3$. If $d_{T}(f, a)=1$, then $d_{T}(f, c)>3$, which is a contradiction. Suppose $d_{T}(f, a)=2$. If $P(f, a)$, the path from $f$ to $a$ in $T$, contains the edge $a b$, then $P(f, a)$ will be of the form $f, b, a$. Now, $\{a, b, c, f\}$ is a $K_{4}$, which is a contradiction. If $P(f, a)$ does not contain the edge $a b$, then $d_{T}(f, c) \geq 4$, which is a contradiction. So, assume that $d_{T}(f, a)=3$. If $P(f, a)$ has no edge in common with $P(b, c)$ and it does not contain the edge ab, then $d_{T}(f, c)>3$, which is a contradiction. So, assume that $P(a, f)=f, x, b, a$. Now, $f, x, b, a$ is a cycle of length 4. So, either $a x \in E(G)$ or $f b \in E(G)$. If $f b \in E(G)$, then $\{a, b, c, f\}$ is a $K_{4}$, which is a contradiction. If $a x \in E(G)$, then $\{a, b, c, x\}$ is a $K_{4}$, which is a contradiction. Next, assume that $d_{T}(b, c)=3$. So, either $P(b, c)=b, x, y, c$ or $P(b, c)=b, a, x, c$. If $P(b, c)=b, x, y, c$, then $d_{T}(a, c)=4$, which is a contradiction. So, $P(b, c)=b, a, x, c$. Now, $d_{T}(a, c)=2$ and $a b \in E(T)$. So, we are in the same situation. (i.e, $d_{T}(b, c)=2, a b \in E(T)$ ). So, using the similar arguments, it can be shown that this situation is not 
possible. Since, $T$ can not contain all the three edges $a b, b c$ and $c a$, exactly two out of these three edges are present in $T$.

\section{Proof of Lemma 3.8}

Proof: Let $x c$ be a semi-strong edge of a tree 3-spanner admissible 2-tree of $G$. So, there exists a triangle $\{x, a, c\}$ which is dependent on a semi-forced triangle $\{a, b, c\}$ having forced edges $a b$ and $b c$. Let $T$ be a tree 3-spanner of $G$. If possible, $x c$ is not an edge of $T$. Since, by Lemma 3.5, $T$ contains at least one edge of $\{a, c, x\}, T$ contains $a x$ as $T$ can not contain $a c$. Since, $M(x c)>1$, there is a triangle $\{x, c, d\}$. Now, $T$ contains an edge of $\{x, c, d\}$. Since, $x c$ is not in $T$, wlg, $c d \in T$. Now, $x, a, b, c, d$ is the unique path in $T$ from $x$ to $d$. So, there is no path in $T$ of length at most 3 from $x$ to $d$. This is a contradiction to the fact that $T$ is tree 3 -spanner. So, $T$ must contain $x c$. Hence, semi-strong edges are forced edges.

Proof of Lemma 3.9

Proof: Since $G$ is a tree 3-spanner admissible 2-tree, $G_{1}$ is also tree 3-spanner admissible 2-tree. Since $\{a, b, c\}$ is an interior triangle of $G$, by lemma 2.4, $G$ has a tree 3-spanner $T^{*}$ containing two of the three edges $a b, b c$, and $c a$. Let $\mathrm{T}=T^{*}-\{e, f\}$. Then, $T$ is a tree 3-spanner of $H_{1}$ containing two of the three edges $a b, b c$ and $c a$.

Part $(\alpha)$ Assume that $\{a, b, d\}$ is an interior triangle in $G_{1}$.

Case 1: $T$ contains $a b$ and $a c$.

Let $T_{1}=\mathrm{T}$ and $T_{2}=(T-\{a c\}) \cup\{b c\}$. Then clearly, $T_{1}$ and $T_{2}$ are the required trees.

Case II: $T$ contains $a b$ and $b c$.

Let $T_{2}=T$ and $T_{1}=(T-\{b c\}) \cup\{a c\}$. Then clearly $T_{1}$ and $T_{2}$ are the required trees.

Case III: $T$ contains $a c$ and $b c$.

Since, $\{a, b, d\}$ is an interior triangle, by lemma $2.4, T$ will contain two of the three edges $a b, a d$ and $b d$. Since $T$ contains $a c$ and $b c, T$ can not contain $a b$. So $T$ has to contain $a b$ and $b d$. Then $a, c, b, d, a$ is a cycle in $T$. This is a contradiction to the fact that $T$ is a tree. Hence, $T$ can not contain ac and bc simultaneously.

So, Lemma 3.9 part $(\alpha)$ is proved. Next, we prove part $(\beta)$.

Part $(\beta)$ Now $\{a, b, d\}$ is not an interior triangle of $G_{1}$ and ab is not a semistrong edge.

case I: $T$ contains $a b$ and $a c$.

Now, $T_{1}=(T-\{a c\}) \cup\{b c\}$ is a tree 3-spanner of $G_{1}$ containing $a b$ and $b c$. So, $G_{1}$ has a tree 3 -spanner containing $a b$ and $b c$. Next, we show that $T$ has a tree 3-spanner containing $a c$ and $b c$. Since, $\{a, b, d\}$ is not an interior triangle, either $a d$ or $b d$ is not developing. Wlg, $b d$ is not developing. If $T$ contains $b d$, then it can not contain $a d$ as it also contains $a b$. So, if $T$ contains $b d$, consider the tree obtained from $T$ by deleting $b d$ and adding $a d$. Clearly, this tree is a 
tree 3-spanner of $G$. So, wlg, assume that $T$ does not contain $b d$. If possible, let $T$ do not contain $d a$. Since, $a b$ is not a semi-strong edge, none of the interior triangle containing ad contains two strong edges. So, if there is an interior triangle containing $d a$, then there will be a tree 3-spanner of $G_{1}$ containing $d a$. So, if possible, assume that there is no interior triangle containing $d a$. Since, $b d$ is not an edge in $T, T$ contains two of the edges of some triangle containing $d a$. Let $T$ contain $a z$ and $z d$ of the triangle $\{a, z, d\}$. Since, none of the triangle containing $d a$ is an interior triangle, either $a z$ or $d z$ is not a developing edge. Wlg, $a z$ is not a developing edge. Now, $(T-\{a z\}) \cup\{d a\}$ is a tree 3-spanner containing the edge $d a$. So, wlg, assume that $T$ contains $d a$. Consider $T_{3}=$ $\mathrm{i}(T-\{a b\}) \cup\{b c\}$. Now we claim that $T_{3}$ is tree 3-spanner of $G_{1}$. Let $x y$ be an edge of $G_{1}$. Let $P(x, y)$ be the path from $x$ to $y$ in $T$. Since $T$ is a tree 3-spanner of $G_{1}$, length $(P(x, y))<4$. If $P(x, y)$ does not contain $a b$, then $P(x, y)$ is also a path in $T_{3}$ of length at most 3 . So assume that $P(x, y)$ contains $a b$. Let $L$ be the length of $P(x, y)$. If $L=1$, then wlg, $x=a$ and $y=b$. Now $P^{\prime}(x, y)=x, c, y$ is a path of length 2 in $T_{3}$. If $L=2$, then wlg, $y=b$. Let $P^{\prime}(x, y)=x, a, c, y$. Then $P^{\prime}(x, y)$ is a path of length 3 in $T_{3}$. Assume that $L=3$. Since $b z$ is not an edge in $T$ for every $z \in V\left(G_{1}\right)-\{a, c, d\}$, ether $x=b$ or $y=b$ or $x, y \in\{e, c\}$. If either $x=b$ or $y=b$ then the other vertex has to be one of $a, d$, or $c$. Since, $b, a, d$ is a path of length 2 in $T_{3},\{x, y\} \neq\{b, d\}$. If $x=b$ and $y=c$, then it is easy to see that $T_{3}$ contains a path of length at most 3 from $x$ to $y$. Hence, $T_{3}$ is a tree 3-spanner of $G_{1}$ containing $a c$ and $b c$. So, if $G_{1}$ has a tree containing $a b$ and $a c$, then it has also tree 3-spanners containing $a b$ and $b c$, and it has a tree 3-spanner containing $a c$ and $b c$.

case II: $T$ contains $a b$ and $b c$.

Now, $T_{1}=(\mathrm{T}-\{\mathrm{bc}\}) \cup\{\mathrm{ac}\}$ is a tree 3-spanner of $G_{1}$ containing ab and ac. So, by case I of (b) above, $G_{1}$ will have a tree 3 -spanner containing ac and bc. case III: $T$ contains $a c$ and $b c$.

Let $T_{1}=(\mathrm{T}-\{\mathrm{bc}\}) \cup\{\mathrm{ab}\}$. It is easy to check that $T_{1}$ is a tree 3-spanner of $G_{1}$.

Hence, $G_{1}$ contains a tree 3 -spanner containing any two of the three edges $\mathrm{ab}, \mathrm{bc}$, and ca.

Proof of Lemma 3.10: We propose a method to construct a tree 3-spanner $T$. Let $T=\phi$. Start with some simplicial edge, say $a b$. Include $a b$ to $T$. Let $\{a, b, c\}$ be the triangle containing $a b$. Since, $G$ has no interior triangle, either $a c$ or $b c$ is not developing. If $G$ has more than 3 vertices, then either $a c$ or $b c$ is a developing edge with respect to the current edge $a b$ and current triangle $\{a, b, c\}$. Wlg, $b c$ is developing. Then add $b c$ to $T$. Now make $b c$ as the current edge and consider all the triangles which are not considered. Let $\{b, c, d\}$ be any triangle containing $b c$. If this is a simplicial triangle, then either add $b d$ or add $c d$ to $T$. If $\{b, c, d\}$ is not a simplicial triangle, then add the developing edge of $\{b, c, d\}$ to $T$. Continue this process till all the triangles are considered. We claim that the tree $T$ so obtained is a tree 3-spanner of $G$. Let $x y$ be an edge 
of $G$. Let $\{x, y, z\}$ be the triangle which was considered earlier by the above method than any other triangle containing $x y$. If $\{x, y, z\}$ is simplicial, then two of the edges of the triangle $\{x, y, z\}$ are included in $T$. Then $d_{T}(x, y)<3$. Suppose, $\{x, y, z\}$ is not a simplicial triangle. If $x y$ is included in $T$, then $d_{T}(x, y)=1$. If it is not included, wlg, $y z$ was the current edge when $\{x, y, z\}$ was considered by the algorithm. Then, $x z$ is a developing edges and so is included in $T$. So, $d_{T}(x, y)<3$. So, $T$ is a tree 3 -spanner of $G$.

Proof of Theorem 3.11:

Proof: Necessity: Necessity follows from Lemma 3.6 and Lemma 3.8.

Sufficiency: We will prove it by induction. Let $n$ be the number of vertices of $G$. For $n=4,5, G$ has at most three triangles. So, $G$ has no interior triangles. Hence, by Lemma 3.10, $G$ contains a tree 3-spanner. Assume that every 2tree having $n$ or fewer vertices without containing a strong triangle has a tree 3-spanner.

Let $G$ be a 2 -tree having $n+1$ vertices and does not have any induced strong triangle. If $G$ has no interior triangle, then by Lemma 3.10, $G$ has a tree 3-spanner. A Assume that $G$ has an interior triangle, say $\{a, b, c\}$.

Let $G_{1}, G_{2}, \ldots, G_{r}, r \geq 3$ be the separated graphs of $G$ with respect to $\{a, b, c\}$. Let $S_{1}=\left\{G_{i} \mid W\left(G_{i}\right)=\{a, b\}\right\}, S_{2}=\left\{G_{i} \mid W\left(G_{i}\right)=\{a, c\}\right\}$, and $S_{3}=$ $\left\{G_{i} \mid W\left(G_{i}\right)=\{b, c\}\right\}$. Note that none of the $S_{i}$ is empty. Since, $G$ does not contain any strong triangle, one of the edges $a b, b c$, and $c a$ is neither a strong edge nor a semi-strong edge. Wlg, $a b$ is neither a strong edge nor a semi-strong edge. So, for all $j, 1 \leq j \leq r, G_{j} \in S_{1}$ implies the principal clique of $G_{j}$, which is a triangle, is not interior. Let $\{b, c, f\}$ and $\{a, c, e\}$ be the principal cliques of $G_{1}$ and $G_{2}$, where $G_{1} \in S_{2}$ and $G_{2} \in S_{3}$.

Case I: Either $\left|S_{i}\right| \geq 2$ for some $i, 1 \leq i \leq 3$ or $\left|V\left(G_{i}\right)-\{a, b, c\}\right| \geq 2 \mid$ for some $i, 1 \leq i \leq r$, where $G_{i}$ belongs to $S_{j}, j=2,3$.

Now, for each $G_{i} \in S_{1}, G^{\prime}=G\left[\{e, f\} \cup V\left(G_{i}\right)\right]$ is a 2- tree without strong triangle with fewer vertices than $G$. So, by induction hypothesis, $G^{\prime}$ is tree 3spanner admissible 2-tree. Now, the set of separated graphs of $G^{\prime}$ with respect to $\{a, b, c\}$ is $\left\{G_{i}, G_{1}^{\prime}, G_{2}^{\prime}\right\}$, where $G_{1}^{\prime}=G[\{a, b, c, e\}]$ and $G_{2}^{\prime}=G[\{a, b, c, f\}]$. So, by Lemma 3.9, each $G_{i} \in S_{1}$ has a tree 3-spanner containing the edges $a c$ and $b c$. By similar arguments and by Lemma 3.9, each $G_{i} \in S_{2}$ and each $G_{j} \in S_{3}$ has a tree 3-spanner containing the edges $a c$ and $b c$. The union of these trees is a tree. This tree is the tree 3-spanner of $G$. Hence, by induction principle, every 2-tree which does not contain any strong triangle as an induced subgraph admits a tree 3 -spanner.

Case II: Each $S_{i}, 1 \leq i \leq 3$ is singleton and $\left|V\left(G_{j}\right)-\{a, b, c\}\right|=1$ for each $G_{j}$ in $S_{i}, i=2,3$.

Consider $G^{\prime}=G\left[\{e, f\} \cup V\left(G_{1}\right)\right], G_{1} \in S_{1}$. Note that, $G^{\prime}=G$. Let $G^{\prime \prime}=G-\{e\}$. Since, $G^{\prime \prime}$ is a 2-tree having $n$ vertices without containing a strong triangle, $G^{\prime \prime}$ is a tree 3-spanner admissible by induction hypothesis. Let $T$ be a tree 3-spanner of $G^{\prime \prime}$. Let $P(a, c)$ be the path from $a$ to $c$ in $T$. Now, length of $P(a, c) \leq 3$. If possible, length of $P(a, c)=3$. Then, $P(a, c)=a, b, f, c$ or $P(a, c)=a, d, f, c$. If $P(a, c)=a, b, f, c$, then $T^{\prime}=(T-\{b f\}) \cup\{b c\}$ is a tree 
3-spanner of $G^{\prime \prime}$ and length of the path $P(a, c)$ in $T^{\prime}$ is 2. Suppose, $P(a, c)=$ $a, d, b, c$. Since, $\{a, d, b\}$ is not an interior triangle, either $b d$ or $a d$ is not a developing edge. Wlg, $b d$ is not a developing edge. Then, $T^{\prime}=T-\{b d\} \cup\{a b\}$ is a tree 3-spanner of $G^{\prime \prime}$ such that there is a path of length 2 from $a$ to $c$ in $T^{\prime}$. So, wlg, $T$ has a path $P(a, c)$ from $a$ to $c$ of length one or two. Now $T \cup\{a e\}$ is a tree 3-spanner of $G^{\prime}$. So, by induction principle, every 2-tree which does not contain a strong triangle as an induced subgraph admits a tree 3 -spanner. 